\title{
Papers
}

\section{Cumulative complexity: Understanding the scope of digital asset liquidity, continuity, and viability}

\begin{abstract}
Ben Howell Davis
is Principal Consultant, Davis International Associates, a digital asset solutions provider focused on strategic planning for long-term digital continuity. Formerly he was Senior Scientist and Strategic Director for Media and Entertainment, Razorfish, Inc.; Manager of Communications, Getty Information Institute, and Manager, Electronic Publications, Getty Trust Publication Services, Los Angeles. Previously he was a research associate at the Massachusetts Institute of Technology, Manager of the AthenaMuse Software Consortium at the Center for Educational Computing Initiatives (MIT/CECI) and Manager of the Project Athena Visual Computing Group. $\mathrm{He}$ was also an instructor at the MIT Media Lab, a Fellow at the MIT Center for Advanced Visual Studies, and a lecturer in the MIT Visual Arts Program. His publications include art and technology reviews for Scientific American Magazine and Time and Bits: Managing Digital Continuity, and When Everything Learns.
\end{abstract}

Keywords: content, production, distribution, intellectual property, continuity, interface, migration, streaming media, cross-platform, metatagging

Abstract With apologies to Karl Marx, there is a specter haunting digital technology. That specter is "cumulative complexity." Finding a way to understand this situation may be useful in illuminating the scope and intensity of the challenges in digital asset management. Operating, acquiring, merging or divesting a digital enterprise requires comprehensive digital asset management at every level all the time.

\section{CUMULATIVE COMPLEX DIGITAL EPHEMERA}

With apologies to Karl Marx, there is a specter haunting digital technology. That specter is "cumulative complexity." We know three things about complexity from current research into complexity theory:

- complexity increases with evolution

- complexity begets complexity

- complexity increases with connectivity.

Digital technology produces evolving complex ephemera. As Gertrude Stein would have described digital "when you get there, there is no there." With digital there are only bits.

Digital production systems are ephemeral, digital content is ephemeral, digital communication is ephemeral, digital storage is ephemeral - digital assets are ephemera.

Digital content is content that is understood at a human level and simultaneously understood and interpreted on the computational level. This is the distinguishing factor of the digital asset. Digital information simultaneously interacts with humans and with computer systems and this conditions begets more complexity.

In addition, digital is evolving as it is being understood. The primary mechanism for this evolution is networking - everything that can communicate will. On the World Wide Web, an electronic publication speaks to the world of humans as well as the world of computer systems. The depth of asset creation and movement is staggering. In digital asset creation novelty becomes the required. The rapid demand for hyperlinks, interactive graphics, full motion video, and sound on the web are all evidence of novel ideas becoming standards.

Digital planning, production, communication, and preservation are now an evolving environmental condition. Current 
methodologies for digital asset management are rooted in experiences in the library and museum communities, the digital solutions and design disciplines, the communication and entertainment industries, and law. Future methodologies may be found in ecology, bioscience, and the cognitive and neurosciences.

Digital assets are created and maintained in support systems architecture, in digital production tools, in digital content development, in taxonomy development, in user destination designs, in audience interactions, and in legal monitoring. Being digital means everything should be accounted for, everything can communicate, everything has value, everything could be reused, and everything could endure. Operating, acquiring, merging or divesting a digital enterprise (and what enterprise isn't digital now?) requires comprehensive digital asset management at every level all the time. What sort of framework for understanding this cumulative complex condition might be useful?

\section{WHAT IS CUMULATIVE DIGITAL COMPLEXITY?}

Cumulative digital complexity arises from three interlinked conditions:

- Digital liquidity refers to how digital assets are created and used. Factors that influence the degree of liquidity are the strategic plans for maximizing a return on the initial investment in digital production, anticipated types of screen destinations like mobile, PC, broadband and/or print, plans for repurposing various types of production, and interface design.

- Digital continuity refers to how digital assets are stored and accessed. The longevity of digital materials is a topic of high interest. Digital technology produces fragments of projects in great quantity. Strategic planning for format conversion, migration, and usage frequency involves understanding and implementing evolving standards, metadata, storage formats, and search/access tools that involve sophisticated common vocabularies. Attention to digital continuity ensures the long-term use of assets.

- Digital viability is concerned with how the use of assets is measured. Increasingly, the digital enterprise must justify budgetary expenditures for liquidity and continuity methods and practices. Tools for measuring return on investment, tools for modeling opportunities, and tools for risk analysis and decision making are beginning to be developed to track the viability of assets into the future.

Enterprises that manage digital liquidity, continuity, and viability create architectures of assets that must be aligned and integrated so that assets communicate on all three levels.

Committing to digital solutions that seamlessly address these concerns is serious, core business practice. Solutions need to be considered for proactive management of digital assets that focus on reducing production costs and creating new revenue opportunities as well as protecting content.

Digital inventories are ever expanding. The growing use of the web, e-commerce, broadband, the proliferation of peripheral media devices, the consumer demand for streamlined access to exciting new forms of content, the increasing deployment of streaming media, the necessity for legal protection of assets, and the quest for new revenue streams and improved workflow efficiency all require deep asset solution strategies.

The focus on efficiencies is especially relevant. Efficiencies maximize production budgets, prevent asset loss or redundant asset production, ensure asset availability for cross-platform distribution, provide security for valuable assets, support partner relationships and provide platform specific access modes for internal or business to business (BtoB) operations. Successful digital alignment and management allows the realization of new revenue opportunities, protects asset rights, tracks asset usage, and allows for incremental asset utilization.

Currently, industry terminology for systems that manage digital liquidity, continuity, and viability are digital asset management (DAM), content management systems (CMS), and digital rights management systems (DRM).

Digital asset management systems digitize physical media assets into storage systems. These systems leverage metadata structures to support asset cataloging and rights licensing policies. Content management systems support digital asset management by providing intelligent access tools to asset storage and archival facilities. Content management systems also enable edit capabilities to support the pre- and post- 
production phases of content development. Digital asset management and content management systems identify most valued assets based on liquidity, reusability, scalability, interoperability, and accessibility. They design asset cataloging and metatagging foundations, digitize and archive priority assets, create core licensing rules, and identify critical legacy system integration initiatives. DAM/CMS validate current core technologies, search features, processes, rules, manage risks of asset loss or redundancies, ensure asset availability for crossplatform distribution, and ultimately optimize user experience.

Digital rights management solutions help solve legal and logistical business problems through asset security and rights protection. Digital rights management solutions control asset usage and enable the collection of usage fees. Digital rights management implements asset tracking, builds order management, enforces access, enables cross-market with partners and enterprise divisions, and bills for asset usage. DRM encourages the exploration and implementation of various tools for securing assets, creates new business models around monetization and digital distribution of content, enables licensing/syndicating content and/or interactive programs, can facilitate wireless/egames (subscription, pay-per-use), creation of custom applications for classroom learning, airlines, and entertainment venues. DRM can facilitate ad-wrapped content, product placement, merchandising, allow for consumer data collection and targeted marketing, implement usage tracking systems, capture user profile information, and monitor and bill for usage.

\section{CUMULATIVE NETWORK ISSUES}

To better understand the scope and depth of the digital asset management condition, a look at network architecture is useful. The technologies that support digital ephemera are network systems. Networked digital technology used for production and distribution is a multi-layered system. There is a standard seven-layer (level) technical model for networked digital technology that describes ways in which lower levels affect higher levels and ways in which higher levels do not (and cannot) know the details of lower levels. ${ }^{1}$

The lower levels provide common functionality that can be used by different implementations of the higher levels. The standard levels are traditionally numbered from the bottom (1) to the top (7), the top being what the system user sees and interacts with. Levels 8-10 are here added to represent additional concerns now apparent in digital asset creation and distribution. Everything starts with the physical (machines, wires), so that is why it is level 1. The model is shown in Figure 1.

Hardware engineers deal with level 1. System engineers commonly speak of working on a layer 2 or 3 problem. Communication engineers deal with levels 4 and 5 . A user or an application developer interacts with the top levels 7-10, which represent access (a login window for instance) to the application (Netscape) and content (electronic book).

The digital production may use application

\begin{tabular}{|c|c|}
\hline 10. & $\begin{array}{l}\text { Distribution: web, warehouse, navigation schemes, strategic design, graphic look and feel, audience } \\
\text { mediation, content administration and maintenance. }\end{array}$ \\
\hline 9. & Content and medium: appropriate media, writing, editing, design, development, production, marketing. \\
\hline 8. & Production application: specific programming for publishing, metadata, conversion from print to electronic. \\
\hline 7. & Application: layer where instructions or requests are received and executed at the operating system level. \\
\hline 6. & Presentation: file transfer protocol program, Netscape browser window. \\
\hline 5. & Session: individual connection to network. \\
\hline 4. & Transport: Transmission Control Protocol (TCP), which works over Internet Protocol (IP). \\
\hline 3. & $\begin{array}{l}\text { Network: includes routing, IP network addresses, and everything else needed to make the internet work no } \\
\text { matter what specific network technologies are at the lower levels. }\end{array}$ \\
\hline 2. & Datalink: includes ethernet protocol or point to point protocol (PPP) over a T-1 line. \\
\hline 1 & Physical: machines, cables, wires, etc. \\
\hline
\end{tabular}

Bottom

Figure 1: Ten layer technical model of networked digital technology 
layer software to display or interact (link) with other material. The electronic author can use application software to write material that is interactive.

The final material, however, has been shaped based on its content, not by arbitrary uses of the lower technology. The electronic author need not technically know how any of the underlying levels work but he/she must be aware of the potentials and limitations of levels $7-10$ in order to make judgments on how to treat content. Authored material is administrated into various schemes for navigation purposes and for visual impact at the distribution level.

The top three (8-10) levels must work in parallel. Content must be developed and seamlessly created for the specific medium in which it will be distributed. Content creators must work directly with application developers and distribution designers. Users interact with content, pass communication back to content creators who interact with applications to apply feedback to improve infrastructure, and so on down the chain. Ultimately something may affect hardware as a final solution to a content reaction - a new scanner is needed because users don't like the quality of a digital image, for instance.

At every level a different form of digital asset is created and will require maintenance over time.

Digital assets are "architected" as well as authored. That is, the content is designed so that it communicates with the computer on whatever level necessary to make the content perform for the user. Adding a link to a text is now a common information architecture on the internet that makes the computer layers perform in such a way as to connect the user to some other system, document, or program and at the same time transport the reader to other content separate from the document he/she is involved with. An electronic article comparing Israeli history with Palestinian history might instruct a person to access related information on British colonialism. An interpreted applet (small, one-function application) would instruct a computer to pass data to a network server to access the information - that server can be anywhere in the world, as can the reader of the article. The digital assets created, updated, and preserved to facilitate this condition are vast.

\section{DIGITAL ASSET COMPLEXITY}

Digital production appears to be shaped by eight forces all of which are necessary to production and distribution. All of these forces seem to be constantly changing and shifting and creating assets:

Concept: information design, the future of words and images in the digital domain. These are the creative factors that drive the initiation, development, and implementation of digital resources. All sorts of planning, prototyping, communication, strategic, and risk factoring assets are created during a concept phase.

Product: editorial, time constraints, physical media limitations such as the capacity of a CDROM/DVD, non-physical media like the web, etc. These are the realities that constrain the form of the final product and create branding and marketing assets as well as the product assets.

Accounting: capital and operating expenses, revenue, online subscription, license fees, budgeting for maintenance of digital material over time, etc. These are the constraints that determine the asset status and future repurposing of digital assets.

Legal: intellectual property such as copyright, trademark, licensing agreements. These are the risk factors inherent in doing business as a digital producer.

Technical: software and hardware required, bandwidth limitations, future opportunities, etc. These are both the opportunities and constraints inherent in free-market technology.

Archival: asset management, preservation, reuse, record keeping, etc. These are the longterm realities of committing assets to a digital form.

Transactional: e-commerce, advertising, promotion, audience data gathering, etc. An ever-evolving electronic marketplace requires participation in order to gain competence.

User: audience, communication, relationship building, marketing, etc. These are the forces of individual choice, mass appeal, and asset value.

All of these forces make some kind of demand on technical infrastructure as well as shape the quality of the product. All of these forces have to be considered when creating and implementing information architecture as well as 
content. The forces must be integrated, both technically and conceptually, for successful production.

An interesting example of levels of cumulative complexity is the history and evolution of the J. Paul Getty Trust's Thesaurus of Art and Architecture $^{2}$ (AAT) from print to electronic publication at the Getty Center in Los Angeles. The Getty Center is an $\$ 8$ bn dollar trust for the arts and culture that includes art object holdings, library materials, conservation technologies, and many philanthropic programs as well as maintaining a \$2bn dollar arts center and museum.

Cumulative reference works like bibliographies and thesauri are naturals for the digital domain because they are works that are essentially never complete. Their contents accumulate from a variety of sources and they are published at regular intervals.

The AAT celebrated its 20th anniversary in 2000. Development of the idea began in 1979-80 and took ten years to bring to press. The thesaurus embraces the cumulative reference concept and collaborative authorship style that make it a natural for digital publication. It collects synonyms for describing works of art and architecture from a variety of international sources, vets the terminology, and establishes standard vocabularies. The decade after its inception witnessed the development of the microcomputer, the proliferation of online databases, and the beginnings of collection management software. This had an effect on the automation of art and architecture collections.

From its beginning, the AAT was thought of as a standard vocabulary tool for the new electronic databases. The notion of a print version was somewhat secondary to the necessity for an electronic version. Researchers and scholars, in describing their work, had clearly articulated the problems:

- the agony of tracking down information when indexes are inadequate

- the difficulty of organizing visual and written information

- the threat of losing information due to lack of certainty of what the information is called in different indexing systems

- the impossibility of finding what you need in unindexed archives. ${ }^{3}$
The AAT first appeared as a three-volume print publication in 1990. In 1992 the Authority Reference Tool (ART) software was created to allow computer access to the data in the first edition. By the time the second edition was published in 1994, ${ }^{2}$ ART was improved to allow immediate access to the data, to make it easy to navigate through the thesaurus, and to make it possible to copy terms from the thesaurus to a database record. This last feature made the AAT a production tool as well as a reference tool.

ART was designed to run on PC-DOS or MS-DOS and was never updated for Windows 95 or Windows NT. Since 1996 it has not been very useful. Since 1997 the AAT has been available as a licensed download from the Getty website without any accompanying software. Licensees incorporate it into their own software applications or have an interface built for it. The AAT was also prototyped as a filtering agent for search engines in 1997-98 and proved very successful as a way to narrow searches on the internet. This last application has carried the AAT from a print publication to computer application to an internet browser to an internet search enhancement. The Art and Architecture Thesaurus is a prime example of the way developments in technology parallel the utility of a cumulative reference literary form. If we apply the forces matrix to the AAT:

Concept: large-scale thesaurus for art and architecture terminology

Product: licensed download

Accounting: capital cumulative asset, licensed

Legal: Getty copyright

Technical: hierarchical text, software potential

Archival: cumulative update that can migrate to any digital media - currently as ftp access

Transactional: nonexclusive license for sliding scale of fees

User: well-established user base with good new user market opportunities - including search engine market, need and information never becomes dated.

The example of AAT is important on many levels. It makes the case that publishing does not mean books per se. Publishing means the shaping of content. Content created to perform in the constraints of the paper page may be freed by digital publishing to become not only new content but a variety of new opportunities as well like search engines. 
This example points to larger issues having to do with production. Digital transmittal, the integration of publication databases, online licensing, conversion of pre-press materials to a variety of formats, issues of digital workflow, archiving, repurposing, dynamic publication catalogs, third-party agreements, and how to establish criteria for deciding what form a publication might take are all concerns that make managing aligning digital assets critical.

\section{ART COLLECTIONS VS INDUSTRY COLLECTIONS}

Another way of illustrating the scope and complexity of digital asset management is to look at the differences between traditional cultural museums and archive and industrial collections. In museums individual objects are of value and increase in value over time, requiring stable long-term systems of asset management. Industry archives are composed of individual objects, groups of objects, production artifacts, and marketing materials that can be of value depending on historic and market demands. Industry archives require variable, flexible, scalable, strategic systems for asset evaluation and management.

An arts organization is unique in that it functions as a school, a museum, a library, a laboratory, and a fiduciary. It is public and private, academic and corporate in its management. It serves both the general public and a variety of highly specialized audiences, from art historians to the general public. It may give grants, sell products, and distribute products freely as a public service. The introduction of networked digital technology into the organization will affect internal and external communications, publishing, record keeping, accounting, financial services, and investing activities. The following partial list offers an example of the complexity and number of computing systems in an arts organization:

- general computers, servers, network and telecommunication systems

- collections management systems

- public kiosks

- visitor reservations system

- contact management and mailing list system

- facilities work order management systems

- human resources management systems
- financial information systems

- library and collection management systems

- specialized database systems

- web browsers

- image collection systems

- subscription system

- publication inventory and fulfillment systems

- bookstore inventory systems

- web e-commerce.

At some point, most of these systems will need to communicate with each other in order to have an efficient means for cataloging, researching, producing, advertising, distributing, archiving, accounting, rights verification, revenue projection, licensing, and communication, both internally and externally. These systems will be the infrastructure of intranet, extranet, for uses with external partners, collaborators, etc, and internet use of digital publications for the general public.

An industry archive will have most of these same asset generating components in addition to an enormous interest in licensing, reuse, marketing, customer relations, and a wide range of potential market interests and future opportunities. The Warner Bros. archive, for instance, holds over 80 million assets - films and all the physical properties that go with them - animation cells, digital production materials, props - the list is obviously enormous. Although the entertainment industry is probably the extreme example it is not difficult to envisage the universe of digital assets and what they represent as capital to a company.

Industry is already beginning to experiment with new forms of payment for distributing digital assets. Change in payment systems will create huge new challenges in the form of "micropayments". ${ }^{4}$ Charges for digital information could start as low as two hundredths $(0.02)$ of a cent, would be a very swift debit/credit transactions at very low transaction cost - a tenth of a cent and up, depending on security, volume, speed - and would be done by automated systems. Micropayments would be necessary in a "disintermediated" system, a system that is direct to the consumer via electronic delivery.

Prices are dependent on transaction costs, the cost of shipping, storage, markup, promotion, advertising, storage as well as production costs 
like editing, markup/typesetting, and server. In the micropayment scenario, transaction costs will drop to virtually nothing and micropayments will allow instantaneous payment, thus taking the billing costs out of the charge. ${ }^{5}$ In the new micropayment option, transaction and intermediation costs plummet, prices become dictated by the nature of publisher, author, and content almost exclusively. In the micropayment world there could be:

- five or ten-year cost recovery timetable, without any significant cost for storage

- continuous-update subscriptions

- fragment fees (0.02) worth per paragraph, section, article, or segment

- variable quality with expense based on how high a resolution is desired

- image with explanatory background for extra cost

- pricing by audience demand

- pricing by audience type

- pricing by time in broad or narrow increments of minutes, days, months

- shareware knowledge

- pricing by knowledge level/filtration/server processing

- $\quad$ pricing by update frequency

- institutional discounts

- association membership discounts.

Standard variables such as content, the audience, the author's demands, and the ongoing costs are now compounded by a potential for a wide variety of consumer desires unique to electronic publishing such as print on demand or usage of portions or fragments of publications. The new demands on the producer may be:

- development of support infrastructure

- explanatory material

- ethical choices regarding prices

- acquisition strategy changes

- rethinking of the "unit" (the book, periodical, etc)

- flexibility of production

- direct relationship with customer

- multiple intermediaries

- subsidiary rights/contractual diversity

- micro-royalty mechanisms.

\section{THE FUTURE}

One area that will be increasingly important to the future of digital asset production is an inventory of intellectual property rights for materials in electronic form. In its broadest implication, an intellectual property assessment generally means determining:

- whether you have all the necessary rights to exploit an item in whatever venue or medium you intend to use it (print, electronic, advertising, performance, etc)

- whether you have taken or can take the necessary steps to adequately protect the item from unlawful use by others in current and future media.

The answers to these questions will depend to a large extent on the way an organization decides to use its intellectual properties. At this point, it is difficult even to estimate the scope or cost of this kind of review without knowing how complex the histories of the various properties are. A complete review might focus on the developers and/or creators of the items, their relationships to the organization at the time the items were acquired or developed (eg, employees, contractors, or contributors, etc) and the sort of agreements they have or had with the organization.

Some initial issue spotting could be done before a definitive plan was implemented. For instance, a sample of properties from the various parts of the organization could be looked into as a way to model the process. The range of intellectual property issues is broad and could include:

- publications

- public affairs materials

- contemporary works

- web materials

- contracts

- licenses

- scholarly works

- agreements

- software

- library materials.

Typically the process for developing successful communication architecture alignment involves intensive strategic planning including an asset inventory, a needs assessment, a thorough understanding of existing systems and practices, and an examination of data collection criteria. 
Planning and preparing reviews of standard vocabularies for describing assets, reviewing standards for continuity and longevity, understanding cross-media definitions, and applying insights into methods and practices are critical. Solutions require defining requirements and alignments and in most cases customization of systems and configurations.

Organizational information systems need to be interoperable, have consistent technical compatibility for future relationships, and develop a core of criteria for judging functionality. Resources for production, development, communication archiving, and accounting — both financial and intellectual property accounting — become increasingly dependent on consistent overall technology architecture. Internal and external uses of digital material have an effect on intellectual property rights, storage and preservation of digital assets, on leveraging advantages vis-à-vis internet use, and on the strategic long-term goals and mission of the organization. The organization's digital library and asset management systems, electronic publishing initiatives, image delivery systems, digital archives, and cumulative digital assets will need to be seen as threads that require careful management. New threads are constantly being added like customer relationship management systems (CRM).

When customer call centers began using software to collect data on each caller, computer telephony integration (CTI) became a business function. The logical extension of this became CRM with the move of the CTI database information back into the organization. Managing relationships with customers is not a new idea but the notion of a level of sales automation with CRM is irresistible. This means that information from the sales force could be retained and potentially used to guide product development. Costs are attached to integrating CRM systems with existing IT systems, aligning information architectures, and creating links to other digital assets, content, and rights management systems. Systems alignment and integration costs can be three to five times initial CRM licensing. CRM has to be thought of strategically rather than as a repository for customer data. CRM is most effective when it wins new customers and retains existing ones. CRM can prompt sales people with information significant to customers' needs and lifestyles including dates and events that personalize the customer experience. Continuing education departments at some universities are beginning to use CRM systems to track what courses students have taken and inform them of new offerings that may be of interest to their career directions. The notion that continuing education is life-long learning makes CRM an attractive tool for retaining students far into the future and keeping enrollment at peak levels. The education consumer is not a new idea for universities but CRM makes it possible to communicate with students in a substantive way that provides the university with data on educational trends while encouraging brand loyalty from customers. The data that these CRM systems generate are invaluable in marketing campaigns and kept over long periods, can reveal potential sources of future revenue as well as guide institutional investment.

\section{THE NEW PAST}

With the advent of digital technology, the great variety of options open to creating content in different forms is constantly expanding. Asset production now includes knowledge of complex digital systems, electronic distribution, industry directions, and vision of future opportunities. This is especially true where highly sophisticated development requires that everyone involved must understand something about every aspect of a digital project — from the mission of the organization to the nature of source material as well as the quality of design, production, distribution, audience, and technology. The client and the service are merged by alignment in digital communication. The continuity of the resulting assets is rapidly becoming a new kind of digital currency.

\section{References}

1 Getty Research Institute (2002) Art and Architecture Thesaurus, J Paul Getty Publications, Los Angeles, CA.

2 Petersen, T. (1994) Introduction to the Art and Architecture Thesaurus, Vol. 1, in The J. Paul Getty Trust Bulletin (Fall 1994): 4-5.

3 Ibid.

4 Jensen, M. (1998) Getty Publications Seminars, Getty Centre, Digital Publishing, Los Angeles. Since Jensen's lecture, ebrary, Inc., an electronic 
publisher associated with Adobe Systems, Inc. has begun (June 2000) to institute the micro-payment concept for accessing online materials by allowing users to copy parts of free documents for a minimum payments of 15 cents per page.

Payment is made with the online equivalent of a phone card.

5 When databases provide human-free billing, the phone bill will drop even further.

\section{Further reading}

Connected: An Internet Encyclopedia, www.freesoft.org/CIE.

Davis, B. H. and MacLean, M. (2000) Time and Bits: Managing Digital Continuity. J. Paul Getty Trust Publications, Los Angeles, CA.

Kauffman, S. A. (1993) The Origins of Order: Self Organization and Selection in Evolution, Oxford University Press, Oxford, UK.

Modis, T. (2003) Forecasting the Growth of Complexity and Change, DUXX Graduate School of Business Leadership, Geneva, Switzerland. 